\title{
Experimental and numerical study of the flow field around a small car
}

\author{
Ivan Dobrev ${ }^{1, *}$, Fawaz Massouh ${ }^{1}$, Amélie Danlos $^{2}$, Michael Todorov ${ }^{3}$ and Plamen Punov ${ }^{3}$ \\ ${ }^{1}$ ENSAM, Dynfluid, 151 bd de L'Hôpital, 75013 Paris, France \\ ${ }^{2}$ CNAM, Equipe Energétique, Turbomachines et Moteurs, Laboratoire Chimie Moléculaire, Génie des Procédés Chimiques et \\ Energétique, CNAM, Saint Martin, 75003 Paris, France \\ ${ }^{3}$ Technical University - Sofia, Faculty of Transport, 8 Kliment Ohridski Blvd., 1000 Sofia, Bulgaria
}

\begin{abstract}
This paper presents the aerodynamic study of a small car, which participated in Shell Ecomarathon Europe competition in the Urban Concept Hydrogen class. The goal is to understand the flow field around the vehicle. First, the flow is studied numerically using computational aerodynamics. The numerical simulation is carried out by means of CFD Fluent in order to obtain the drag force experienced by the vehicle and also the flow field. Then the flow field around the car is studied in a wind tunnel by means of particle image velocimetry (PIV). The comparison of the flow fields obtained numerically and experimentally shows good correspondence. The obtained results are very helpful for future car development and permit to improve the drag and to obtain a good stability.
\end{abstract}

\section{Introduction}

Vehicle aerodynamics is very important and has a significant impact on its general performance, especially on fuel consumption. A brief overview of the history of vehicle aerodynamics is presented in [1]. There exist different aerodynamic problems as drag reduction, vehicle stability at high speed, engine cooling, etc. However, the primary issue is drag reduction and many authors studied the drag sources and presented different methods for reducing it [1-3]. It should be noted that the field of vehicle aerodynamics is very large, has different tasks depending on car type and it is difficult to summarize all existing problems. In this paper, the authors focus their research on the aerodynamics of small car, which participated in Shell Eco-marathon Europe competition in the Urban Concept Hydrogen class. What is specific about this kind of vehicle is the low Reynolds number due to the low speed. The shape of the car is not optional but must observe the rules of the competition [4].

The aerodynamics of a small car to participate in the Shell Eco-marathon has been studied by a limited number of researchers. At first glance it seems that aerodynamic forces can be neglected due to the low vehicle speed. However, these forces are comparable with rolling resistance forces, because of the low vehicle weight. Therefore, the aerodynamic study is important for obtaining the best vehicle performance.

The authors of paper [5] present a fluid simulation of a solar prototype vehicle employing $\mathrm{k}-\varepsilon$ turbulence model. The results of simulations give satisfactory results for drag coefficients, which is by $8,2 \%$ less than the experimental value. The analysis of energy losses shows that the aerodynamic losses are $41 \%$ of all loses for this class of vehicle and the authors conclude that the losses depend primarily on rolling resistance and aerodynamic drag, even in the case when the speed does not exceed $38 \mathrm{~km} / \mathrm{h}$.

The authors in [6] simulated a flow around a small electric car for urban mobility at the stage of preliminary design. They studied the grid dependency of the solution and showed that the drag coefficient of 0,129 varies by less than $3 \%$ when the nodes increase from nearly 0,65 million to 2,4 million.

The paper [7] presents a study which is useful for the design of new vehicles for incoming races. The set of simulations was prepared for several Shell Eco Marathon models for a range of velocities from 20 to $45 \mathrm{~km} / \mathrm{h}$. The results of the simulations show a strong dependence of the aerodynamic drag on the Reynolds number. The authors demonstrate how flow simulation can contribute to the optimization of vehicle shape. Contrary to [5] they show that the aerodynamics drag is $50 \%$ of the rolling resistance but here the drag coefficient of 0,09 is much lower.

The author in [8] presents the development of the shape of a light road vehicle by means of flow simulation with Navier-Stokes and Boltzmann lattices solvers. The aerodynamic optimization is done by design evolution from low-drag body to the final vehicle design together with the aesthetic control of the shape after each change. The simulation is carried out on grid up to 11 million nodes with different turbulent models. The author reports that the lift coefficient shows a big change compared to the drag when the solver or turbulent model is changed.

* Corresponding author: ivan.dobrev@ensam.eu 
The aim of this work is to simulate the flow around an existing model in order to obtain the aerodynamic drag and lift forces and also the flow field around the vehicle. Then this flow field will be compared with the real flow obtained in the wind tunnel by means of particle image velocimetry (PIV). It must be noted that the flow field around the vehicle and the pressure distribution are in close relationship with the aerodynamic drag. The velocity field shows the separation, which is very useful for understanding aerodynamic drag and makes it possible to study the impact of the vehicle shape on the drag. Hence this study will be useful for further amendments of vehicle shape for future races.

\section{Numerical simulations}

\subsection{Numerical model}

The flow around the vehicle with a length of $3,180 \mathrm{~m}$, a width of 1,210 and a height of 1,010 m is modelled by means of commercial Navier-Stokes solver ANSYS Fluent 15. The computational domain is a box that includes the vehicle and has dimensions of $50 \mathrm{~m} \times 12 \mathrm{~m}$ $\times 12 \mathrm{~m}$ (length $\times$ width $\times$ height). Such dimensions allow for the reduction of the boundary effects on the solution. On the upstream, top and side walls of this domain we apply the boundary condition of type "velocity inlet" because the flow is not perturbed by the vehicle. In this simulation the vehicle is fixed and in order to represent its movement, the velocity applied on all "velocity inlet" walls is equal to the desired vehicle speed.

As the vehicle wake perturbs the downstream so the boundary condition of the downstream wall must be of "pressure outlet" type. In order to represent the movement of the vehicle with respect to the ground and the wheels rotation, we apply Multi Reference Frame model (MRF). In this model the wall which represents the ground is moved respectively to the vehicle with the same velocity which is applied on walls with "velocity inlet" boundary condition. Also the wheels are supposed to be rotating around their axles with the angular velocity which corresponds to the desired vehicle speed.

The computational domain has a mesh size of approximately 20 million polyhedral nodes and 5 prism layers on the wall of the vehicle and also of the ground. The height of the first prism layer of $0,05 \mathrm{~mm}$ ensures $\mathrm{y}+<5$, which is sufficient for this kind of computation. The calculation is unsteady, with time step of $0,01 \mathrm{~s}$ and 10 iterations per time step. The applied turbulence model is hybrid - Delayed Detached Eddy Simulation (DDES), [9]. This DDES model significantly improves the computation where flow separation occurs as in the tested case [9]. However, the calculation becomes power and memory consuming and requires a few days' calculation on a computer with 2 processors with 8 cores and a RAM memory of $64 \mathrm{~Gb}$. In this study it is assumed that the solution converges if for a given averaging period, the drag and lift forces become relatively constant, which is achieved usually after 1500 time steps.

\subsection{Numerical results and analysis}

The first computation is carried out for vehicle speed of $10 \mathrm{~m} / \mathrm{s}$. The lift and drag coefficients are obtained by dividing the lift $L$ and $\operatorname{drag} D$ forces by the upstream dynamic pressure and the vehicle cross section $S$ :

$$
\begin{aligned}
& C_{D}=\frac{D}{\frac{\rho V^{2}}{2} S} ; \\
& C_{L}=\frac{L}{\frac{\rho V^{2}}{2} S} .
\end{aligned}
$$

Here $\rho$ is air density of $1,225 \mathrm{~kg} / \mathrm{m}^{2}, V$ is upstream velocity of $10 \mathrm{~m} / \mathrm{s}$ and $S$ is cross section of vehicle of $0,67283 \mathrm{~m}^{2}$. The obtained drag coefficient is equal to 0,1614 , which is typical for such kind of vehicle. The lift coefficient of $-0,238$ is negative which permits the increase of the vehicle stability. The flow field in the vehicle symmetry plane is presented in Fig. 1.

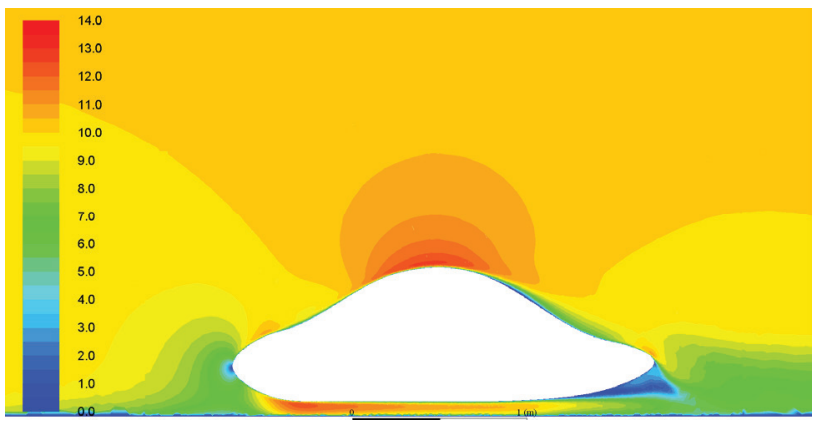

Fig. 1. Field of velocity $V$ in $\mathrm{m} / \mathrm{s}$.

At the front end of the car, the flow between the car underside and the ground is accelerated. This acceleration is due to the high position of the front end. The rear end of the vehicle is also high, so the flow separates from underside where the recirculation zone occurs. However, the flow is unnecessarily accelerated at the upper side of the rear end, Fig. 2.

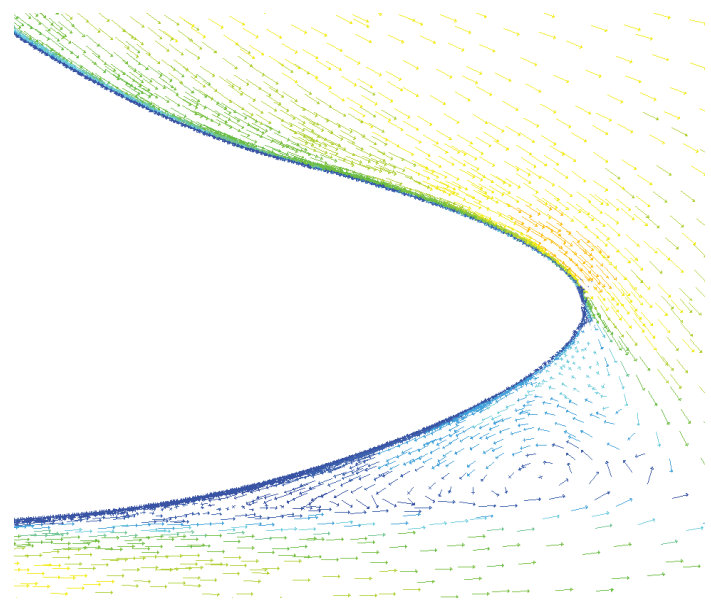

Fig. 2. Velocity vector at the rear end. 
The velocity field in the horizontal plane at a distance of $0,3 \mathrm{~m}$ from the ground is presented in Fig. 3. At the front end of the vehicle, the flow is unnecessarily accelerated due to the high curvature near the front wheel. On the contrary, the rear end angle of the vehicle is very important and the flow detaches from the lateral sides. The detachment is not steady and its position varies periodically with time similarly to wake behind a bluff body.

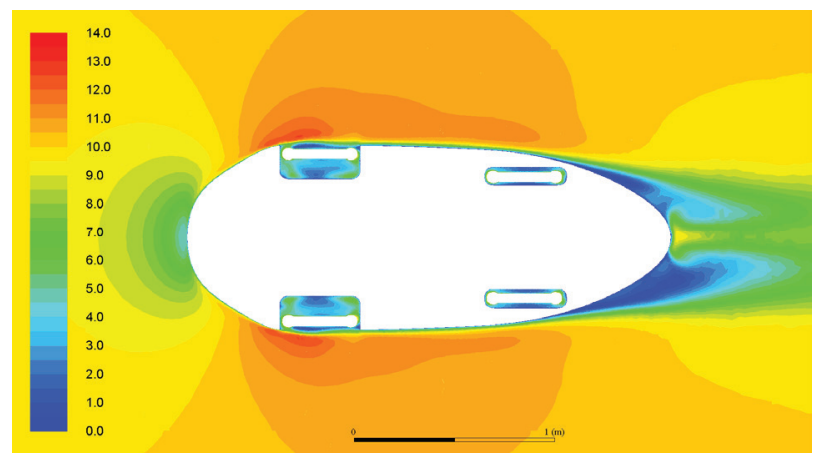

Fig. 3. Velocity vector at the rear end.

The wake behind the vehicle is represented by means of iso-vorticity surfaces, Fig. 4. The vortex wake shows also that the flow is detached from the rear lateral sides. In this image the vehicle surface is coloured by means of static pressure. The lowest pressure which is represented by deep blue colour corresponds to high velocity and permits to visualise the flow acceleration at the front wheels.

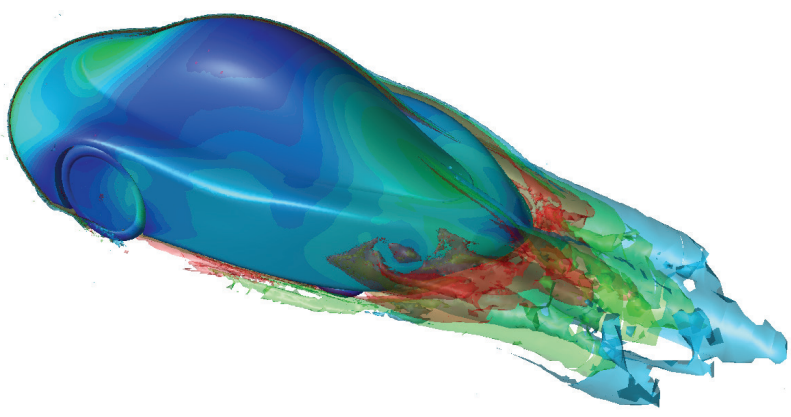

Fig. 4. Vortex wake behind the car.

In this study, for the forces and PIV measurements, we use a vehicle model with a length of $0,19 \mathrm{~m}$, which is quite small compared with a real vehicle. In addition, the ground of the test bench is fixed, therefore the relative movement between the vehicle and the ground cannot be represented. For this reason a second simulation is needed with a Reynolds number corresponding to wind tunnel experiment. To simplify the solution, compared to previous case, only fluid viscosity is changed and the ground and the wheels are fixed. As can be expected the drag coefficient is by $24 \%$ bigger and equal to 0,2 . The lift coefficient becomes now positive and equal to 0,038 . Such a big change can be explained by the decrease of the flow velocity between the vehicle underside and the ground.

\section{Experimental study}

\subsection{Force measurements}

The experimental study is conducted in the wind tunnel of Dynfluid Laboratory at ENSAM-Paris. This wind tunnel is a Prandtl type (closed-circuit) and is equipped with a three-blade fan with a rotor diameter of $3 \mathrm{~m}$. The flow is homogenized in a settling chamber by means of honeycomb strengtheners and wire mesh. The nozzle contraction ratio is 12,5 , which permits to obtain uniform velocity profile with a turbulence level lower than $0,25 \%$. The test section is semi-guided and the flow is not constrained laterally, which allows for an increase in the dimensions of the tested models. The test section has a cross-section of $1,35 \mathrm{~m} \times 1,65 \mathrm{~m}$ and a length of $2 \mathrm{~m}$. Its static pressure is equal to the atmospheric pressure and the upstream velocity depends only on the stagnation pressure in the settling chamber. During the experiments the tunnel velocity is measured by a differential pressure transducer connected with the settling chamber and the atmosphere. The fan is driven by a frequency-controlled asynchronous motor, which permits a change in the wind tunnel velocity from $1,8 \mathrm{~m} / \mathrm{s}$ up to $40 \mathrm{~m} / \mathrm{s}$.

The wind tunnel is equipped with two superposed balances linked to each other. The first balance is a sixcomponent and can measure up to $1500 \mathrm{~N}$ with a precision of $0,075 \mathrm{~N}$. The second balance can measure only four components: lift, drag, pitch and roll. This balance has a precision of $0,01 \mathrm{~N}$ in the interval of $0 \mathrm{~N}$ to $50 \mathrm{~N}$. The strain gauges of the balances are connected to a data acquisition system HBM MGCPlus, which is equipped with high quality strain gauges amplifiers and analog to digital converters. The acquisition system is controlled by means of PC with a specially developed Labview program.

The studied vehicle model with a length of $0,19 \mathrm{~m}$ is placed on the second balance by means of rectangular flat plate with dimensions of $0,5 \mathrm{~m} \times 0,2 \mathrm{~m}$. This plate represents the ground. The measurements for upstream velocity of $25 \mathrm{~m} / \mathrm{s}$ are conducted during a time interval of $60 \mathrm{~s}$ with a sampling rating of $10 \mathrm{~Hz}$. For such low forces as in the present case study, the drag coefficient can be determined with a precision of $5 \%$. The averaged drag coefficient is 0,24 , which is $20 \%$ bigger than the simulation value. Such discrepancy can be justified because the solver has difficulties in taking into account the viscosity effects for a low Reynolds number. Additionally, the boundary layer is completely turbulent without any laminar part as it exists in reality.

\subsection{PIV measurements}

The velocity around the vehicle model is obtained by means of particle image velocimetry (PIV). This method is non-intrusive and does not perturb the flow. The flow is seeded by special mist generator which permits to visualise the flow. The method of PIV is based on crosscorrelation between two successive images which permits to calculate the particle displacement. As this displacement is low, the shutter speed is very high and 
the camera needs high intensity light which is ensured by the flash of impulse laser. Specially dedicated software ensures the synchronisation between the PIV camera, the frame grabber and the impulse laser: The principles of PIV are explained in details in [10].

In this exploration the PIV system is controlled by DynamicsStudio 2015a of Dantec. The images are taken with a Nd-Yag Litron Nano-L 200-15, with a power impulse of $200 \mathrm{~mJ}$ and a Dantec FlowSense 4M camera with a resolution of $2048 \times 2048 \mathrm{px}$. The camera is equipped with AF Micro-Nikkor $60 \mathrm{~mm} \mathrm{f/2,8D} \mathrm{lenses.}$ The synchronization system is equipped with NI-1428 frame-grabber card to synchronize the images with laser flashes. The flow is seeded with micro-droplets of olive oil generated by a mist generator Dantec 10F03. The average droplet diameter is $2-5 \mu \mathrm{m}$.

The wind tunnel velocity during the exploration is $13,7 \mathrm{~m} / \mathrm{s}$. To obtain good averaging the totality of 500 double images are taken. A typical raw image is presented in Fig. 5 where it is possible to observe the small olive droplets illuminated by the laser. Additionally, it can be observed that the flow underside is not illuminated therefore the velocity vectors cannot be calculated for this area.

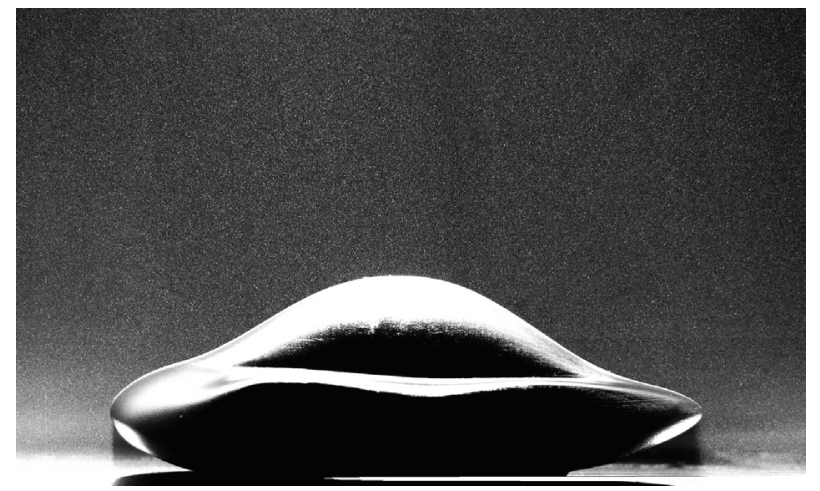

Fig. 5. PIV raw image.

To calculate the PIV velocity in this study we apply Adaptive PIV method, which permits to obtain a spatial resolution of $1,5 \mathrm{~mm}$ with good precision. The flow which is obtained by averaging the 300 snapshot is presented in Fig. 6.

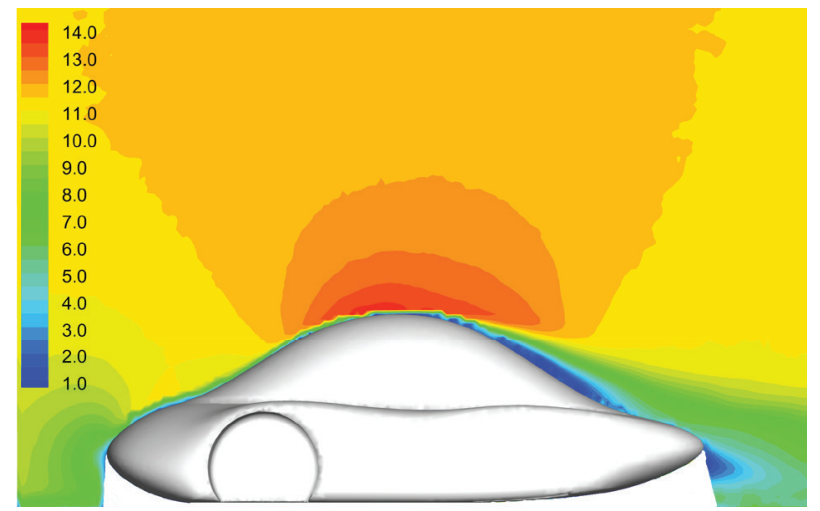

Fig. 6. Averaged velocity field obtained by means of PIV.
Generally, the simulation result presented in Fig. 1 is similar to the experimental velocity field apart from the flow behind the canopy, which is slightly separated.

\section{Conclusions}

This numerical and experimental study concerns the aerodynamics of a vehicle which participated in Shell Eco-marathon. The objective is to obtain drag and lift coefficients and also the flow field around the car. The presented numerical study makes it possible to obtain the aerodynamic performance of the real vehicle and also of the small vehicle model, which is used for wind tunnel exploration. The lift coefficient varies moderately due to the influence of Reynolds number while the lift exhibits strong variation due to the additional influence of ground fixing.

Finally, the small vehicle model is tested in the wind tunnel for obtaining the drag and the flow field in the plane of symmetry. In general, the results of the comparisons between the experiments and the simulation are satisfactory. The numerical simulations reveal the recirculation zones which should be avoided. The study shows that CFD modeling will be useful for future design of the vehicle.

\section{References}

1. W. Hucho, Aerodynamics of road vehicles: from fluid mechanics to vehicle engineering (Elsevier, 2013)

2. J. Katz, Race car aerodynamics: designing for speed (R. Bentley 2006)

3. S. McBeath, Competition Car Aerodynamics: A Practical Handbook (Veloce Publishing House 2015)

4. http://www.shell.com/energy-and-innovation/shellecomarathon.html

5. G. Galmarini, S. Dell'Agostino, M. Gobbi, G. Mastinu, Solar Prototype for Shell-Eco Marathon Race (No. 2017-01-1260) SAE Technical Paper (2017)

6. Abo-Serie, E. Oran, O. Utcu, Aerodynamics consideration for low drag Shell Eco-Marathon competition car, ICAME-2016, Istanbul, Turkey (2016)

7. A. Cieslinski, W. Prym, M. Stajuda, D. Witkowski, Investigation on Aerodynamics of Super-Effective Car for Drag Reduction Mechanics and Mechanical Eng., 20(3), 295-308, (2016)

8. D. Cogan, The aerodynamic design and development of an urban concept vehicle through CFD analysis, Diss, Cape Peninsula Univ. of Tech. (2016)

9. P. Spalart, Detached-eddy simulation, Ann. Rev. of fluid mech., 41, 181-202, (2009).

10. M. Raffel, C. Willert, S. Wereley, J. Kompenhans, Particle image velocimetry: a practical guide, Springer. (2013) 